\title{
Photo-catalytic Degradation of Chlorendic Acid: 1. Degradation Products
}

\section{NDOKIARI BOISA (Ph.D.)}

\author{
Department of Chemistry, Faculty of Science, Rivers State University of Science and Technology, P.M.B. 5080, \\ Nkpolu, Port Harcourt, Nigeria
}

\begin{abstract}
The photo-catalytic degradation of chlorendic acid in the presence of $\mathrm{TiO}_{2}$ was conducted to investigate the reaction pathways and possible degradation products as a means of assessing the environmental legacies of polyesters that are designed with both chlorendic acid and $\mathrm{TiO}_{2}$ chemicals. Comparison of liquid chromatography/mass spectrometry spectra of chlorendic acid and degradation solutions suggests $\mathrm{HCl}, \mathrm{H}_{2} \mathrm{O}$ and chlorendic acid anhydride as the main products of the degradation process. @JASEM
\end{abstract}

\section{http://dx.doi.org/10.4314/jasem.v17i3.1}

Chlorendic acid $\left(\mathrm{C}_{9} \mathrm{H}_{4} \mathrm{Cl}_{6} \mathrm{O}_{4}\right)$ (CAS No. 115-28-6) (Figure 1) is used as a chemical intermediate in the manufacture of polyester resins, polyurethane foams, wool fabric alkyd resin paints, oligoesters, piping and engineering plastics, to impart flame retardant property (HSDB, 2013). The acid is also used as an extreme pressure lubricant and in the synthesis of functional metal organic frameworks (MOFs) (HSDB, 2013; Cui et al., 2013). Recently studies have been conducted to investigate how the incorporation of both chlorendic acid and $\mathrm{TiO}_{2}$ into the framework of polymers can improve optical stability, stiffness, chemical inertness and flame retandancy (e.g. Morrion, 2004; Laachachi et al., 2005; Ghosh, 2006; and Laachachi et al., 2006). Due to its industrial applications chlorendic acid is released to the environment during industrial processes or leached from polyester products at dump sites (HSDB, 2013; NTP, 2011). Consequently it is imperative to investigate the behavior of chlorendic acid in different environmental matrices in the presence of $\mathrm{TiO}_{2}$.

Chlorendic acid is inert to biological degradation (Sebastian et al., 1996). In aquatic ecosystems it is inhibitory to algal and microfaunal activity (Hendrix et al., 1983). For human population chlorendic acid is an anticipated carcinogen and can irritate the skin, eye and respiratory tract (HSDB, 2013). Based on the anticipated impact of chlorendic acid on both aquatic systems and human population studies have been conducted to identify efficient protocols for its degradation.

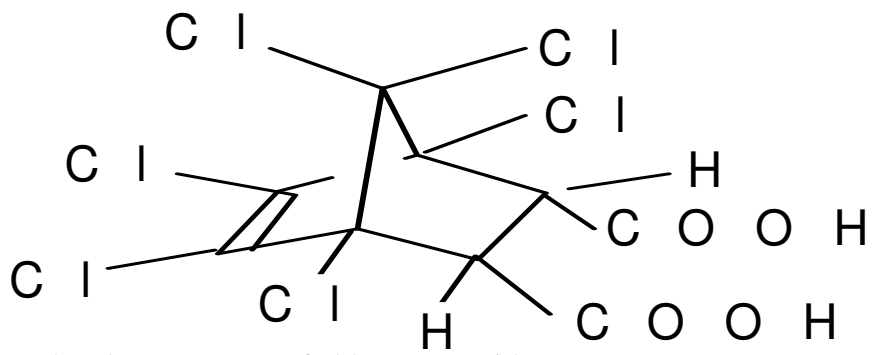

Fig1: Molecular structures of chlorenaic acid.

Mutation, single phase ozonation, two phase ozonation, heating, and pyrolysis have been investigated for the degradation of pure chlorendic acid in solutions or chlorendic acid in the framework of esters (Kai et al., 1991; Stowell and Jensen, 1991; Sebastian et al., 1996; Freshour et al., 1996, Mullen et al., 1997; RujKumar et al., 2008; Rajkumar et al., 2012). However, concerns have been raised about the use of organic solvents and the lack of data on possible degradation products. Thermal degradation products of chlorendic acid incorporated within polymer frameworks (e.g. oligoesters) have been extensively investigated by RajKumar et al., (2008) and RajKumar et al (2012) but data on degradation products of the leachate form in the presence of $\mathrm{TiO}_{2}$ additive is not available. Titanium dioxide has been explored as a catalyst for the degradation of organic pollutants from water (Hoffman et al., 2003; Jiang et al., 2007; Hidaka et al., 2008; Oncescu et al., 2010; Einaga et al 2013). At atmospheric pressure aqueous suspension of $\mathrm{TiO}_{2}$ under the influence of Ultraviolet (UV) irradiation generates hydroxyl radical (HO') 
that initiates the degradation process (Marci et al., 2003). The present work aims to simulate the degradation of chlorendic acid in water bodies under UV radiation in the presence of $\mathrm{TiO}_{2}$ nanoparticles and identify possible degradation pathways and by products.

Experimental: Titanium dioxide photocatalyst (98\% purity) was purchased from BDH limited with. Chlorendic acid (99\% purity) was purchased from Aldrich logistic GmbH, Germany and used as received. A laboratory built photo-reactor (figure 2) consisting of two 80 watt Philip Low-pressure UV lamps (UVA 315-400 nm), 4000ml Pyrex beaker, a magnetic stirrer and an oxygen inlet source was employed as the reaction cell. Duplicate aqueous suspensions of chlorendic acid [200 ml: $(100 \mathrm{mg} / \mathrm{L})$ and $\left.\mathrm{TiO}_{2}(1.0 \mathrm{~g})\right]$ were placed in $400 \mathrm{ml}$ Pyrex beaker and positioned in the photo-reactor cell for 50mins, under UV-irradiation in the presence of oxygen. At the end of the reaction portions of suspension were extracted with pipette, centrifuged (Centaur MSE centrifuge instrument) for 15 mins at 2700 RPM. Small portions of the resulting supernatant were siphoned with a syringe and filtered through Whatman syringe filter (Whatman filter paper pore size: $0.2 \mu \mathrm{m}$ ) to remove $\mathrm{TiO}_{2}$ particle still suspended in the supernatant. The clear liquids obtained from the control and degraded suspensions were submitted to Liquid Chromatography-Mass spectrometry (LCQ Advantage - Thermo Finngan) equipment for analysis. The MS detection was performed with atmospheric pressure/Matrix Assisted Laser Desorption Ionization (AP/MALDI) in negative mode. A Jenway $3010 \mathrm{pH}$ meter with a 662-1759 probe was used for the $\mathrm{pH}$ measurement of aliquots extracted during photodegradation reaction.

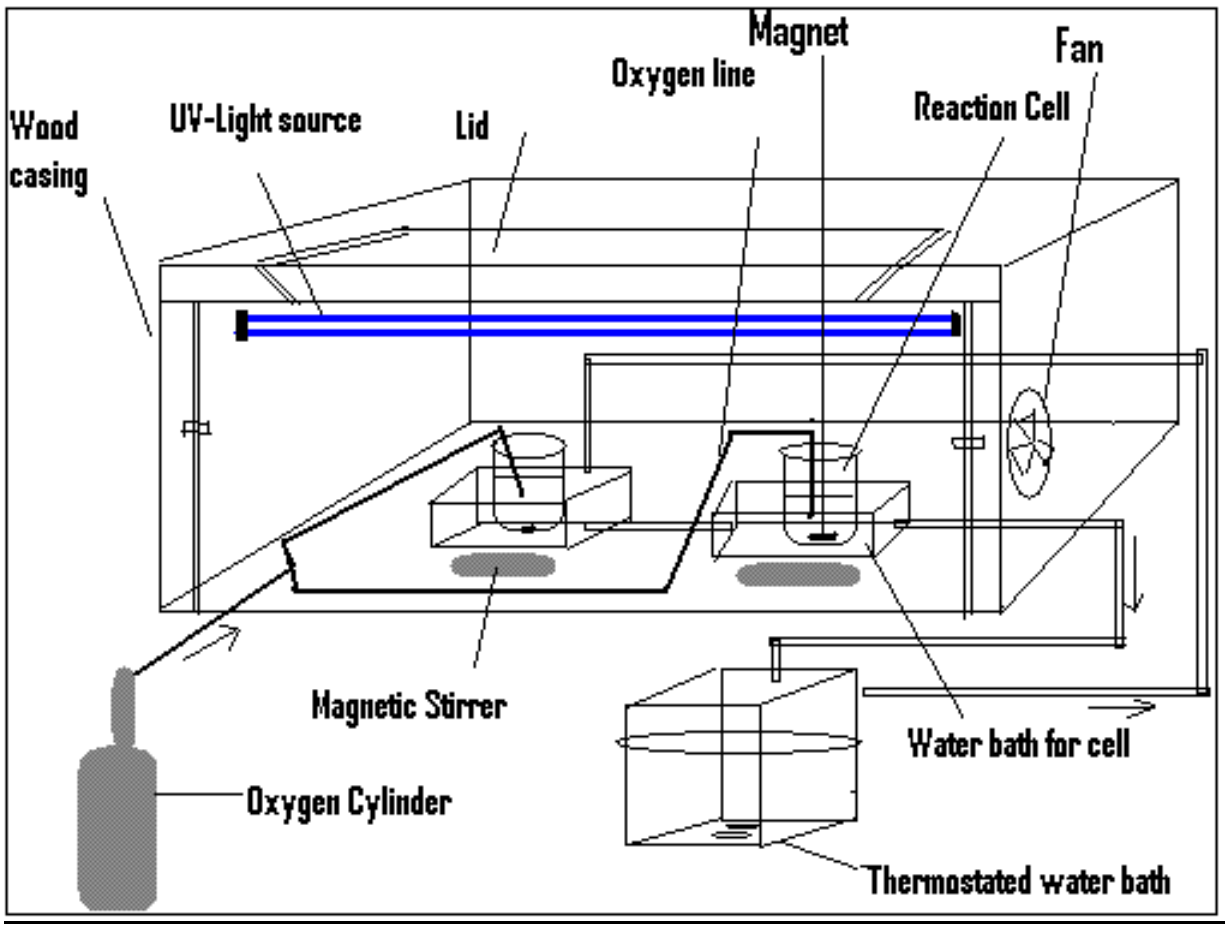

Fig 2: Scheme of the Laboratory built photo-reactor cell

\section{RESULTS AND DISCUSSION}

Spectra of the LC-MS analyses of both the control (chlorendic acid) and photo-catalytically degraded chlorendic acid solutions are provided in figures 4 and 5 respectively. Comparison of the spectra for control solution and photo-catalytically degraded solution indicates enhancement of peaks at 351.2 and 279.5. The comparison also indicates new peak at $251.4,369.3$ and 444.8 for the photo-catalytically degraded chlorendic acid solution. The $\mathrm{m} / \mathrm{z}$ values at $279.5,351.2$ and 369.3 in the mass spectra for the degraded chlorendic acid solution may be due the loss of two molecule of $\mathrm{HCl}(\mathrm{M}-36)$, one molecule of $\mathrm{HCl}(\mathrm{M}-36)$ and one molecule of $\mathrm{H}_{2} \mathrm{O}$ (M-18) from chlorendic acid, respectively. The products $\mathrm{HCl}$ and $\mathrm{H}_{2} \mathrm{O}$ suggested in this work were also reported as thermal degradation products of chlorendic acid based oligoesters by Rajkumar et al., (2008). After degradation the $\mathrm{pH}$ value of the extracted aliquot dropped from 3.00 to 2.23 and the lower $\mathrm{pH}$ value could be due the in situ incorporation of $\mathrm{HCl}$ during the photocatalytic degradation process.

\section{NDOKIARI BOISA (Ph.D.)}


The $\mathrm{m} / \mathrm{z}=369$ have previously been observed during the pyrolysis of unsaturated polyester resin based on chlorendic acid (Vijayakumar et al., 1983; Riajkumar et al., 2008). The molecular peak at 369.3 represents chlorendic acid anhydride which may have resulted from the dehydration of chlorendic acid. Vijayakumar and Lederer, (1990) and Mullen et al. (1997) have previously identified chlorendic acid anhydride as a product of the pyrolysis of chlorendic acid based polyesters. The in situ conversion of chlorendic acid to the anhydride may have been facilitated by the formation $\mathrm{HCl}$ during the degradation since previous studies used mineral acids to catalyze ring closure (e.g. Omuaru and Boisa, 1994)

The prominence of the (M-36) peak on the spectra of degraded sample and the lower $\mathrm{pH}$ value suggest that the degradation of chlorendic acid involves dehydrochlorination instead of the previously reported (Kai et al., 1991; Sebastian et al., 1996) dechlorination route. Previous analysis of pyrolysis products of chlorendic acid based polyesters indicated the presence of pentachloro- and tetrachloro derivatives (Irzl et al., 1987). The peak at 444.8 may be an ester of chlorendic acid and is consistent with the formation of esters of chlorendic acid during pyrolysis (Irzl et al., 1987). The scheme (Figure 4) is therefore proposed as a preliminary reaction pathway.

The inclusion of $\mathrm{TiO}_{2}$ in chlorendic acid based polyester framework to improve flame ratardancy, optical and other physical properties may also facilitate its conversion to chlorendic acid anhydride in water bodies under sunlight as suggested in Figure 4. The conversion to the anhydride may alter the toxicity of chlorendic acid based polyester products because chlorendic acid anhydride has been associated with allergic respiratory diseases (Hlaskoski et al., 2009). According to the International Programme on Chemical Safety (IPCS) report (IPCS, 1996) chlorendic acid anhydride is suggested to converts easily to chlorendic acid when in water but results from this study indicate that in the presence $\mathrm{TiO}_{2}$ and UV irradiation the process is reversed. Therefore, the suggestion that chlorendic acid anhydride in water will always be hydrolyzed to chlorendic may have been over generalized. Based on the data from this study it appears the toxicity of chlorendic acid anhydride in fluvial environments may have been underestimated.

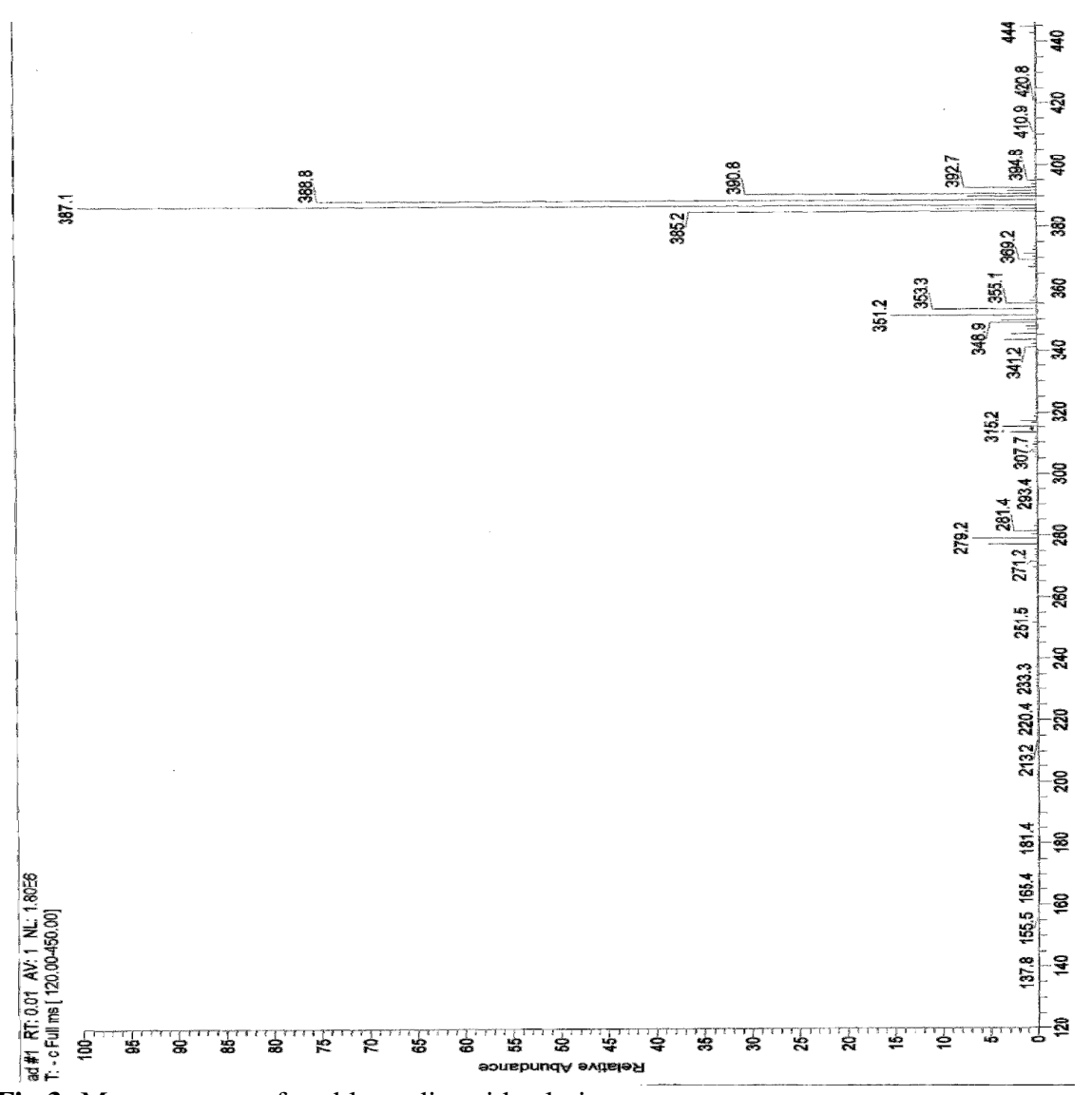

Fig 3: Mass spectrum for chlorendic acid solution

\section{NDOKIARI BOISA (Ph.D.)}




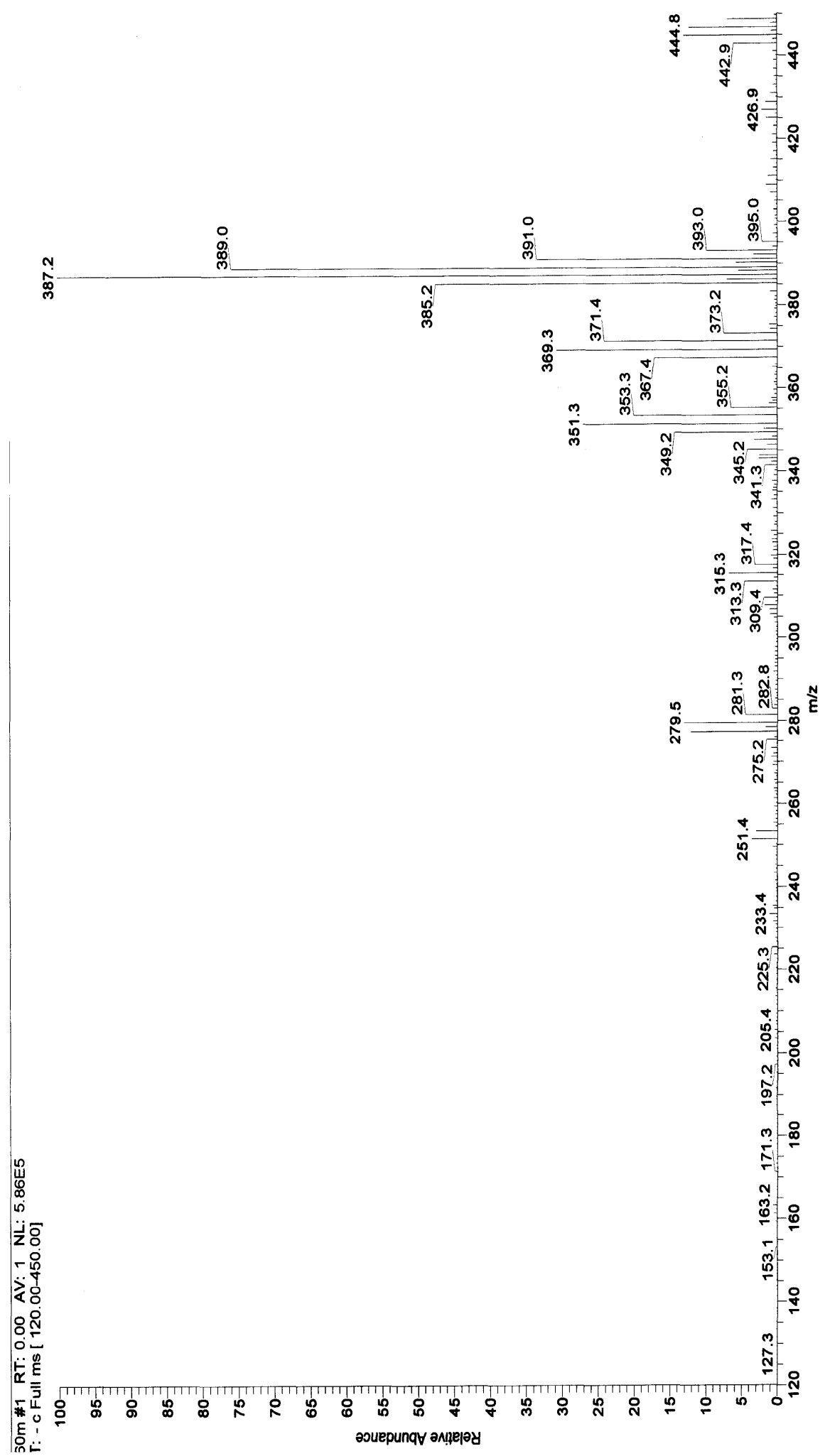

Fig 4: Mass spectrum for photo-catalytically degraded chlorendic acid solution 


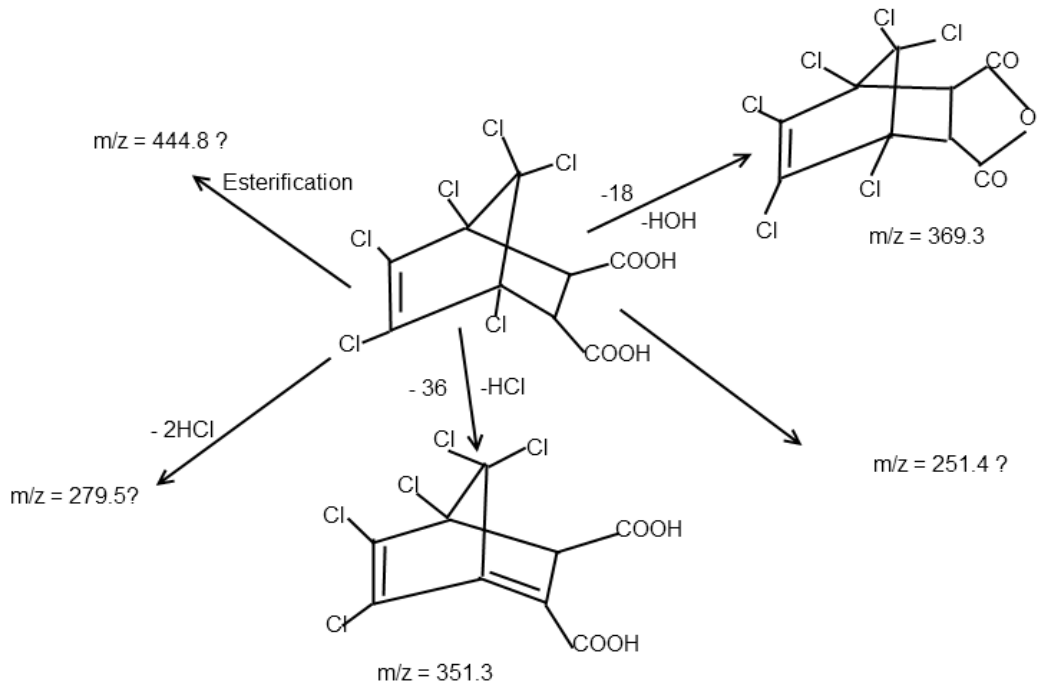

Fig 5: Proposed $\mathrm{TiO}_{2}$ assisted photocatalytic degradation scheme

Conclusion : The photocatalytic degradation of chlorendic acid in aqueous solution was studied using $\mathrm{TiO}_{2}$ as a catalyst. Comparison of LC-MS mass spectra of chlorendic acid and degradation solutions suggest the formation of $\mathrm{HCl}, \mathrm{H}_{2} \mathrm{O}$ and chlorendic acid anhydride. The degradation was mainly via dehydrochlorination and dehydration pathways. Since chlorendic acid anhydride formed during the degradation process is considered as irritating to the eye, skin and respiratory tract there is need to reevaluate toxicity of polymers containing chlorendic acid and $\mathrm{TiO}_{2}$ in their frameworks.

Acknowlegements- The author would like to acknowledge Rivers State Government for the sponsorship of the author during his study at the Northumbria University, UK.

\section{REFERENCES}

Bard, A.J (1979). Photoelectrochemistry and heterogeneous photo-catalysis at semiconductors. Journal of Photochemistry 10: 59-75

Cui, P-P; Cui, L-F; Zhang, L-L; Sun, D-F (2013). Halogen bonding in the assembly of a 1D cadmium (II) polymer based on chlorendic acid (HET). Zeitschrift fur anorgaische und allgemeieneChemie (in press DOI: 101002/zaac.201300141).

Einaga, H; Mochiduka, K; Teraoka, Y (2013). Photocatalytic oxidation processes for toluene

\section{NDOKIARI BOISA (Ph.D.)}

oxidation of $\mathrm{TiO} 2$ catalysis. Catalysis 3: 219231.

Freshour, A.R., Mawhinney, S., and Bhattacharyya, D., (1996). Two phase ozonation of hazardous organics in single and multicomponent systems. Water Research 30: 1949-1954

Ghosh, S.K (2006). Functional Coatings by Polymer Microencapsulation. WILEY-VCH Verlag GmbH \& Co. KGaA, Weinhein, Germany.

Hendrix P.F; Hamala J.A; Langner C.L; Kollig H.P (1983). Effect of chlorendic acid, a priority toxic substance on laboratory aquatic ecosystems. Chemosphere 12: 1083-1099.

Hidaka, H; Garcia-Lopez, E; Palmisano, L; Serpone, N (2008). Photoassisted mineralization of aromatic and aliphatic $\mathrm{N}$-heterocycles in aqueous titanium dioxide suspensions and fate of nitrogen heteroatoms. Applied Catalysis B: Environmental 78: 139-150

Hlaskoski, E; Kuuliala, O; Aalto-Korte, K (2009). Occupation contact urticarial caused by cyclic acid anhydrides. Contact Dermatitis 60: 214-221.

Hoffmann, M.R; Martin, S.T; Choi, W; Bahnemann, D.W (1995). Environmental applications of semiconductor photocatalysis. Chemical Reviews 95: 69-96

HSDB. 2013. Hazardous Substances Data Bank. National Library of Medicine. 
http://toxnet.nlm.nih.gov/cgi-bin/sis/search. Last accessed: 5/2013.

IARC. Monographs on the evaluation of carnogenic risk to humans. Overall evaluation of carcinogenicity: an updating of IARC monographs 1-42, suppl, 7, Lyon: International Agency for Research on Cancer 1994:21

International Programme on Chemical Safety (IPCS) (1996) Environmental Health Criteria 185. Chlorendic acid and anhydride. World Health Organization (WHO), Geneva. http://www.inchem.org/documents/ehc/ehc/ehc1 85.htm\#SectionNumber:7.5

Irzl, G.H; Vijayakumar, C.T; Fink, J.K; Lederer, K (1987). A comparative study of the thermal degradation of unsaturated polyester resins containing various chlorinated norbornene dicarboxylic acid units in the backbone. Journal of Analytical and Applied Pyrolysis 11: 277-286.

Jiang, D; Zhang, S; and Zhao, H (2007). Photocatalytic degradation characteristics of different organic compounds at $\mathrm{TiO} 2$ nanoporous film electrodes with mixed anatase/rutile phases. Environmental Sciences \& Technology 41: 303-308.

Kai G; Weber A.S; Ying W.C (1991). Use of continuous-flow UV-induced mutation technique to enhance chlorinated organic biodegration. Journal of Industrial Microbiology 8: 99-106.

Laachachi, A; Cochez, M; Leroy, E; Gaudon, P; Ferriol, M; Lopez Cuesta, J.M (2006). Effect of $\mathrm{Al}_{2} \mathrm{O}_{3}$ and $\mathrm{TiO}_{2}$ nanoparticles and APP on thermal stability and flame retardance of PMMA. Polymer for Advance Technologies 17: 327-334.

Laachachi, A; Leroy, E; Cochez, M; Ferriol, M; Lopez Cuesta, J.M (2005). Use of oxide nanoparticles and organoclay to improve the thermal stability and fire retardancy of poly (methyl metacrylate). Polymer Degradation and Stability 89: 344-352.

Marci, G; Addamo, M; Augugliaro, V; Coluccia, S; Garcia-Lopez, E; Loddo, V; Martra, G; Palmisano, L; Schiavello, M (2003). Photocatalytic oxidation of toluene on irradiated $\mathrm{TiO} 2$ : comparison of degradation performance in humified air, in water and in water containing a zwitterionic surfactant. Journal of Photochemistry and Photobiology A: Chemistry 160: $105-114$
Morrion, A.R (2004). The Chemistry and Physics of Coatings. The Royal Society of Chemistry, Thomas Graham House, Science Park, Milton Road, Cambridge, U.K.

Mullens, J; Reggerrs, G; Ruysen, M; Carleer, R; Yperman, D; Franco, D; Van Poucke, L.C (1997). The determination of the gases released during heating of a flame retardant for polymers coupling of TG with FTIR, MS and GC-MS. Journal of Thermal Analysis 49: 1061-1067.

National Toxicity Programme (NTP). Report on carcinogens, twelfth edition (2011), http://ntp.niehs.nih.gov/ntp/roc/twelfth/profiles/ ChlorendicAcid.pdf last accessed 5/2013.

Omuaru, V.O.T: Boisa, N (1994). 1-Benzazepine: Part 1- synthesis of novel tetracyclic and pentacylic azepines from a Diels-Alder adducts. Indian Journal of Chemistry 34B: 336-337

Oncescu, T; Stefan, M.I; Oancea, P (2010). Photocalytic degradation of dichlorvos in aqueous $\mathrm{TiO} 2$ suspensions. Environmental Science and Pollution Research 17: 1158-1166

Rajkumar, T; Vijakumar, C.T; Sivasamy, P; and Wilkie, C.A (2008). HET acid based oligoestersTGA/FTIR studies. European Polymer Journal 44: 1865-1873.

Rajkumar, T; Sivasamy, P; Sreedhar, B; Vijayakumar, C.T (2012). Chlorinated thermal stabilizer for optically clear PMMA. Polymer Advanced Technologies 23: 829.

Sebastian, J.H; Weber, A.S; Jensen, J.N (1996). Sequential chemical/biological oxidation of chlorendic acid. Water Research 30: 1833-1843.

Stowell J.P; Jensen J.N (1991). Dechlorination of chlorendic acid with ozone. Water Research 25: 83-90.

Vijayakumar, C.T; Fink, J.K (1983). Flame cooling action of HEX ejected from HET moiety. Thermochimica Acta 60: 225-227

Vijayakumar, C.T; Lederer, K (1990). An insight into the degradation aspect of $1,4,5,6,7,7-$ hexachlorobicyclo[2.2.1]hept-5-ene-2,3dicarboxylic acid based two component polyesters. Thermochimica Acta 173: 129-135.

\section{NDOKIARI BOISA (Ph.D.)}

